\title{
Decomposing the queue length distribution of processor-sharing models into queue lengths of permanent customer queues
}

\author{
Sing-Kong Cheung ${ }^{\mathrm{a}, *}$, Hans van den Berg ${ }^{\mathrm{b}, \mathrm{c}}$, Richard J. Boucherie ${ }^{\mathrm{a}}$ \\ ${ }^{a}$ University of Twente, Department of Applied Mathematics, Stochastic Operations Research Group, \\ P.O. Box 217, 7500 AE Enschede, The Netherlands \\ ${ }^{\mathrm{b}}$ TNO Information and Communication Technology, P.O. Box 5050, 2600 GB Delft, The Netherlands \\ ${ }^{c}$ University of Twente, Department of Computer Science, Design and Analysis of Communication Systems, \\ P.O. Box 217, 7500 AE Enschede, The Netherlands
}

Available online 10 August 2005

\begin{abstract}
We obtain a decomposition result for the steady state queue length distribution in egalitarian processor-sharing (PS) models. In particular, for multi-class egalitarian PS queues, we show that the marginal queue length distribution for each class equals the queue length distribution of an equivalent single class PS model with a random number of permanent customers. Similarly, the mean sojourn time (conditioned on the initial service requirement) for each class can be obtained by conditioning on the number of permanent customers. The decomposition result implies linear relations between the marginal queue length probabilities, which also hold for other PS models such as the egalitarian PS models with state-dependent system capacity that only depends on the total number of customers in the system. Based on the exact decomposition result for egalitarian PS queues, we propose a similar decomposition for discriminatory processor-sharing (DPS) models, and numerically show that the approximation is accurate for moderate differences in service weights.
\end{abstract}

(C) 2005 Elsevier B.V. All rights reserved.

Keywords: Processor-sharing queues; Queue length; Decomposition; Permanent customers; Approximation; Generalized discriminatory processor-sharing

\footnotetext{
* Corresponding author.

E-mail addresses: S.K.Cheung@utwente.nl (S.-K. Cheung), J.L.vandenBerg@telecom.tno.nl (H. van den Berg), R.J.Boucherie@utwente.nl (R.J. Boucherie)
} 


\section{Introduction}

The processor-sharing (PS) service discipline is of considerable interest in many application areas in which different users receive a share of a scarce common system resource. In particular, in the field of the performance evaluation of computer and communication systems, the PS discipline has been widely adopted as a convenient paradigm for modeling bandwidth sharing.

Kleinrock [16] introduced the simplest and best known egalitarian PS discipline, in which a single server assigns each customer a fraction $1 / n$ of the service capacity when $n>0$ customers are in the system. In particular, he showed that the mean sojourn time conditional on the initial service requirement $x>0$ is proportional to $x$. For an extensive body of literature on (egalitarian) PS queues, we refer to Yashkov's survey paper [20].

Cohen [10] generalized the standard PS model into a PS model in which each customer receives a service rate according to an arbitrary positive function $\phi(n)$. By appropriate choice of $\phi(n)$, this model describes a very wide class of service disciplines, and significantly enhances the modeling capabilities of the standard PS model. In many practical applications it models the main factors determining the performance, while on the other hand, it is simple enough to be analytically tractable (see, e.g., [4,17]).

Another generalization of PS is the discriminatory processor-sharing (DPS) discipline, where a customer of type $k$ receives service rate $w_{k} / \sum_{j=1}^{K} w_{j} n_{j}$, according to the set of weights $\left\{w_{j}: j=1, \ldots, K\right\}$ and when $n_{j}$ customers of type $j$ are present in the system. If all weights $w_{j}$ are equal, then we have the ordinary PS queue. Under DPS it is possible to give preferential treatment (non-preemptive) to one or more customer classes at the expense of others. The range of applications for DPS is extremely large (see, e.g., [1,6,9,14]). Exact analysis of DPS turns out to be difficult compared to ordinary PS. Therefore, results for DPS are scarce in the literature. Most notably, the simple geometric queue length distribution for the ordinary PS queue does not have a counterpart for DPS, and tractable transform results for the sojourn time distribution seem not to exist.

For DPS, Fayolle et al. [11] showed that the conditional mean sojourn times satisfy a system of integro-differential equations and derived closed-form expressions for the case of exponential service requirements. In that case, the unconditional sojourn times can be obtained from a system of linear equations. Rege and Sengupta [19] obtained the moments of the queue length distributions as the solutions to linear equations for the case of exponential service requirements, and they also proved a heavytraffic limit theorem for the joint queue length distribution. These results were extended to phase type distributions by van Kessel et al. [13]. Kim and Kim [15] found the moments of the sojourn time in the M/M/1 DPS queue as a solution of linear simultaneous equations. More recently, Avrachenkov et al. [2] showed that the mean queue lengths of all classes are finite under the usual stability condition, regardless of the higher moments of the service requirements. They also showed that the conditional sojourn times of the different customer classes are stochastically ordered according to the DPS weights.

In the present paper, for multi-class egalitarian and discriminatory PS models we investigate a decomposition of the marginal queue length distributions into the queue length distributions of PS models with permanent customers. In particular, for the egalitarian PS model we obtain an exact and analytically tractable decomposition that is remarkable and interesting on its own and offers additional insights into egalitarian PS queues. We apply this decomposition to discriminatory PS to obtain an efficient and analytically tractable approximation of the queue length distribution and mean sojourn times.

More specifically, for a two-class egalitarian PS queue with Poisson arrivals $\lambda_{1}, \lambda_{2}$, when $\left(N_{1}, N_{2}\right)$ is the joint steady state queue length, we show that the marginal queue length $N_{i}$ is in distribution equal 
to $\tilde{N}_{i}^{\left(N_{j}+1\right) *}$, with $i \neq j$. The latter random variable denoted as $\tilde{N}_{i}^{\left(N_{j}+1\right) *}$ can be interpreted as the steady state queue length of a single class M/G/1 PS queue with arrival rate $\lambda_{i}$ and with a random number of permanent customers distributed as $N_{j}$ (i.e., marginal queue length of the other type in the original two-class PS queue).

The decomposition result implies that the marginal queue length distribution for class 1 , factorizes over the number of class 2 customers, and where the factorizing coefficients are equal to the queue length probabilities of an isolated PS queue for type 1, given that type 2 customers are permanent in the system. This queue length decomposition result can be generalized for arbitrary number of classes $K$, and similar results hold for other egalitarian PS models, e.g., PS networks with feedback customers, and PS models with state-dependent but balanced class capacities, which are treated in Section 3.

In Section 4, we propose an approximation method for DPS models based on the queue length decomposition result. The basic assumption is that an isolated customer class in DPS is considered to behave like an egalitarian PS model with reduced capacity and a random environment that is exogenously determined. More specifically, if one type of customers is treated as permanent in a general two-class DPS model, then the model is analytically tractable for the non-permanent class, with reduced service capacity that is exogenously given. The approximations are obtained as solutions of linear systems.

\section{Model}

In this section we introduce a general single server processor-sharing model with $K$ customer classes and we introduce the notation used in this paper. Customers arrive at a single server according to individual and independent Poisson processes with rate $\lambda_{k}>0$ for customer class $k, k=1, \ldots, K$. The service requirements of type $k$ customers are i.i.d. random variables with a general distribution $F_{k}(x)=\mathbb{P}\left(X_{k} \leq x\right)$ with mean $\mathbb{E} X_{k}$. Denote the load of class $k$ by $\rho_{k}=\lambda_{k} \mathbb{E} X_{k}$, and the total offered load by $\rho:=\sum_{j=1}^{K} \rho_{j}$. The server shares its capacity among all customers present in the system. Denote $\mathbf{n}=\left(n_{1}, \ldots, n_{K}\right)$ as the system state with $n_{j}$ customers of type $j$. The server capacity may be dependent on the system state. When the system state is $\mathbf{n}$, the total rate class $k$ receives is $\phi_{k}(\mathbf{n})$. All customers within class $k$ share the capacity $\phi_{k}(\mathbf{n})$ in an egalitarian manner, i.e., each customer in class $k$ receives rate $\phi_{k}(\mathbf{n}) / n_{k}$. We assume that $\phi_{k}(\mathbf{n})=0$ if and only if $n_{k}=0$. The total server capacity is denoted by $\phi(\mathbf{n}):=\sum_{k=1}^{K} \phi_{k}(\mathbf{n})$.

This general model describes a very wide class of service disciplines. In particular, it includes the following special cases of processor-sharing models:

(a) Egalitarian processor-sharing (with fixed capacity): $\phi_{k}(\mathbf{n})=n_{k} / \sum_{j=1}^{K} n_{j}$.

(b) Egalitarian processor-sharing with state-dependent service capacity: $\phi_{k}(\mathbf{n})=n_{k} \phi(\mathbf{n}) / \sum_{j=1}^{K} n_{j}$. Note that in the original generalized PS model studied by Cohen [10], $\phi(\mathbf{n})$ only depends on $\mathbf{n}$ through its sum $n_{1}+\cdots+n_{K}$.

(c) Discriminatory processor-sharing (with fixed capacity): $\phi_{k}(\mathbf{n})=w_{k} n_{k} / \sum_{j=1}^{K} w_{j} n_{j}$.

This model framework also covers DPS models with state-dependent service capacity $\phi(\mathbf{n})$ and state-dependent service weights $w_{k}(\mathbf{n})$. The DPS models with both state-dependent capacity and statedependent weights are called generalized discriminatory processor-sharing (GDPS) models in [9]. 
The egalitarian PS models a. and b. are analytically tractable (when $\phi(\mathbf{n})$ only depends on $\mathbf{n}$ through its sum $n_{1}+\cdots+n_{K}$ ). In particular, analytical expressions are available for the equilibrium distributions of customers simultaneously present in the system (and marginal distributions), mean number of customers $\mathbb{E} N_{k}$ of class $k$, mean sojourn time $\mathbb{E} T_{k}$ and conditional mean sojourn time $\mathbb{E} T_{k}(x)$ of a class $k$ customer given its initial service requirement $x>0$. For GDPS models, these expressions have not yet been obtained in tractable form.

\section{Decomposition of egalitarian processor-sharing models}

In this section, we first establish decomposition results for the ordinary egalitarian PS model. Results for more general egalitarian PS models are briefly indicated at the end of this section.

Consider an egalitarian processor-sharing model with two types of customers (indexed by $l=1,2$ ), class capacity functions $\phi_{l}(\mathbf{n}):=\phi_{l}\left(n_{1}, n_{2}\right)=n_{l} /\left(n_{1}+n_{2}\right)$, and where the second class of customers is possibly an aggregate of several other classes. Let the random vector $\left(N_{1}, N_{2}\right)$ denote the joint steady state queue length of this processor-sharing model; its distribution has the product form (cf. [10,12]):

$$
\mathbb{P}\left(N_{1}=i ; N_{2}=j\right)=(1-\rho)\left(\begin{array}{c}
i+j \\
j
\end{array}\right) \rho_{1}^{i} \rho_{2}^{j},
$$

when the stability condition is satisfied, i.e., $\rho:=\rho_{1}+\rho_{2}<1$, and is insensitive to the service requirement distributions apart from their means (see, e.g., [7]). For $i, j \in \mathbb{Z}_{+}$, the marginal queue length probabilities are given by

$$
\mathbb{P}\left(N_{1}=i\right)=\frac{1-\rho}{1-\rho_{2}}\left(\frac{\rho_{1}}{1-\rho_{2}}\right)^{i}, \quad \mathbb{P}\left(N_{2}=j\right)=\frac{1-\rho}{1-\rho_{1}}\left(\frac{\rho_{2}}{1-\rho_{1}}\right)^{j} .
$$

\subsection{Queue length decomposition}

Theorem 1 shows how the marginal steady state queue length probabilities of the two-class PS queue can be related through the negative binomial probabilities $\alpha(i, j)$ and $\beta(j, i)$, defined as

$$
\begin{aligned}
& \alpha(i, j):=\mathbb{P}\left(\tilde{N}_{1}^{(j+1) *}=i\right)=\left(1-\rho_{1}\right)^{j+1}\left(\begin{array}{c}
i+j \\
i
\end{array}\right) \rho_{1}^{i}, \\
& \beta(j, i):=\mathbb{P}\left(\tilde{N}_{2}^{(i+1) *}=j\right)=\left(1-\rho_{2}\right)^{i+1}\left(\begin{array}{c}
i+j \\
j
\end{array}\right) \rho_{2}^{j},
\end{aligned}
$$

$\sum_{i=0}^{\infty} \alpha(i, j)=1$ for all $j \in \mathbb{Z}_{+}, \sum_{j=0}^{\infty} \beta(j, i)=1$ for all $i \in \mathbb{Z}_{+}$, where $\tilde{N}_{k}$ denotes the steady state queue length of an isolated $\mathrm{M} / \mathrm{G} / 1$ PS queue with arrival rate $\lambda_{k}$ and general service requirement distribution $F_{k}(x)$. The random variable denoted as $\tilde{N}_{k}^{m *}$ has a distribution which is the $m$-fold convolution of the distribution of $\tilde{N}_{k}$. Assume that $\tilde{N}_{i}$ is independent of $N_{j}$, for $i \neq j$.

Theorem 1. For $i, j=1,2$ and $i \neq j$, the marginal queue length $N_{i}$ is in distribution equal to the random variable $\tilde{N}_{i}^{\left(N_{j}+1\right) *}$, i.e., $N_{i} \stackrel{d}{=} \tilde{N}_{i}^{\left(N_{j}+1\right) *}$, where $\tilde{N}_{i}^{\left(N_{j}+1\right) *}:=\sum_{m=0}^{N_{j}} \tilde{N}_{i, m}$, with $\left\{\tilde{N}_{i, m}\right\}_{m \geq 0}$ i.i.d. and distributed as $\tilde{N}_{i}$. 
Proof. First observe that the following equality holds by combining Eqs. (1)-(4):

$$
\mathbb{P}\left(N_{1}=i ; N_{2}=j\right)=\alpha(i, j) \mathbb{P}\left(N_{2}=j\right)=\beta(j, i) \mathbb{P}\left(N_{1}=i\right) .
$$

Hence, by conditioning on $\left\{N_{2}=j\right\}$ and independence of $\tilde{N}_{1}$ and $N_{2}$, we have for all $i \in \mathbb{Z}_{+}$:

$$
\mathbb{P}\left(\tilde{N}_{1}^{\left(N_{2}+1\right) *}=i\right)=\sum_{j=0}^{\infty} \alpha(i, j) \mathbb{P}\left(N_{2}=j\right)=\mathbb{P}\left(N_{1}=i\right),
$$

i.e., $\tilde{N}_{1}^{\left(N_{2}+1\right) *} \stackrel{d}{=} N_{1}$. Analogously, we have that $\tilde{N}_{2}^{\left(N_{1}+1\right) *} \stackrel{d}{=} N_{2}$.

Corollary 2. From Theorem 1, we obtain the following set of linear equations:

$$
\mathbb{P}\left(N_{1}=i\right)=\sum_{j=0}^{\infty} \alpha(i, j) \mathbb{P}\left(N_{2}=j\right), \quad \mathbb{P}\left(N_{2}=j\right)=\sum_{i=0}^{\infty} \beta(j, i) \mathbb{P}\left(N_{1}=i\right) .
$$

The decomposition theorem can be generalized for arbitrary number of classes $K$, and also for multi-class egalitarian PS models with state-dependent service capacity that only depends on the total number of customers present in the system (see Section 3.4).

Theorem 1 can be interpreted as follows. In the two-class PS model, the marginal queue length $N_{1}$ is in distribution equal to a queue length from a related M/G/1 queue with permanent customers. The latter M/G/1 queue has additional $j$ permanent customers with probability $\mathbb{P}\left(N_{2}=j\right)$. To this end, note that the queue length distribution in an ordinary M/G/1 PS queue with $j$ permanent customers, equals the $(j+1)$-fold convolution of the queue length distribution of the same model without permanent customers (see [5]).

The remarkable fact is that $a(i, j)=\mathbb{P}\left(\tilde{N}_{1}=i \mid j\right.$ permanent customers) and $\mathbb{P}\left(N_{1}=i \mid N_{2}=j\right)=$ $\mathbb{P}\left(N_{1}=i ; N_{2}=j\right) / \mathbb{P}\left(N_{2}=j\right)$ are identical and independent of $\rho_{2}$, i.e., the 'conditional steady state probability' of the original two-class PS queue equals the 'single class permanent customer' probability $a(i, j)$.

From the class 1 point-of-view in the original two-class PS model, it seems as if class 1 behaves according to an ordinary single class $\mathrm{M}_{\lambda_{1}} / \mathrm{G} / 1$ PS queue with $j$ permanent customers if $j$ customers of type 2 are present in the system. Furthermore, if there is a customer arrival (resp. departure) for type 2 in the system, then it seems as if class 1 instantaneously 'jumps' to a $\mathrm{M}_{\lambda_{1}} / \mathrm{G} / 1$ model with $j+1$ (resp. $j-1$ ) permanent customers, and as if the new equilibrium (steady state behavior) is instantaneously attained at the jump epoch.

\subsection{Sojourn time decomposition}

After establishing the queue length decomposition result, a natural question is whether or not a similar decomposition result holds for the sojourn time distribution. It can be shown that a similar decomposition holds for the first moment of the conditional sojourn time distribution (see Theorem 3), where $T_{k}(x)$ is the sojourn time for customer type $k$ with initial service requirement $x>0$ in the original two-class PS model, and $\tilde{T}_{i}^{\left(N_{j}+1\right) *}(x)$ is the conditional sojourn time of the isolated M/G/1 PS queue with arrival rate $\lambda_{i}$ and a random number of permanent customers $N_{j}$. 
Theorem 3. For all $x \geq 0$, the conditional mean sojourn times can be decomposed into

$$
\begin{aligned}
& \mathbb{E} T_{1}(x)=\mathbb{E}\left(\tilde{T}_{1}^{\left(N_{2}+1\right) *}(x)\right) \equiv \sum_{j=0}^{\infty} \frac{(j+1) x}{1-\rho_{1}} \mathbb{P}\left(N_{2}=j\right), \\
& \mathbb{E} T_{2}(x)=\mathbb{E}\left(\tilde{T}_{2}^{\left(N_{1}+1\right) *}(x)\right) \equiv \sum_{i=0}^{\infty} \frac{(i+1) x}{1-\rho_{2}} \mathbb{P}\left(N_{1}=i\right),
\end{aligned}
$$

where $(m+1) x /\left(1-\rho_{k}\right)$ is the mean conditional sojourn time of an isolated M/G/I PS queue with arrival rate $\lambda_{k}$, service requirement distribution $F_{k}(x)$ and with $m$ permanent customers.

Proof. From Eqs. (2), (5) and (6), it is readily verified that $\mathbb{E}\left(\tilde{T}_{1}^{\left(N_{2}+1\right) *}(x)\right)=\mathbb{E}\left(\tilde{T}_{2}^{\left(N_{1}+1\right) *}(x)\right)=x /(1-\rho)$, which is the same as the well-known result $\mathbb{E} T_{1}(x)=\mathbb{E} T_{2}(x)=x /(1-\rho)$.

Obviously, the result for unconditional mean sojourn times is similar; since it also follows directly from the exact decomposition result for queue length distributions and Little's law. For higher moments of the (conditional) sojourn time distribution, it can be easily seen that a similar decomposition does not hold in general. However, if both customer classes have the same service requirement distribution, then a certain stochastic ordering result has been proven in [8]. More specifically, the moment ordering:

$$
\mathbb{E}\left(\tilde{T}_{1}^{\left(N_{2}+1\right) *}(x)\right)^{k} \leq \mathbb{E}\left(\tilde{T}_{2}^{\left(N_{1}+1\right) *}(x)\right)^{k}
$$

holds for all $x \geq 0$ and $k \geq 2, k \in \mathbb{N}$, if and only if $\lambda_{1} \geq \lambda_{2}$. From this moment ordering result, insensitive upper bounds for all moments of the sojourn time distribution (conditioned on the initial service requirement) for an ordinary M/G/1 PS queue are obtained. In addition, these upper bounds have a very special structure with so-called Eulerian numbers in the expressions (see [8]).

\subsection{A feedback network with egalitarian processor-sharing}

Now consider a processor-sharing network with an egalitarian PS node and a node used by a single feedback customer. Exogenous customer arrivals at the PS node form a Poisson process with rate $\lambda>0$, and these customers are served at the PS node with i.i.d. service requirements; generally distributed with mean $\mathbb{E} X$. The service requirement for the feedback customer at the PS node is generally distributed and denoted by the random variable $Z$. After service completion of the feedback customer at the PS node, the feedback customer is routed to the feedback node (with probability 1) where he spends a generally distributed time $Y$. After this random time $Y$ at the feedback node, the feedback customer joins the PS node for a service requirement $Z$.

If we denote $\mathbb{P}\left(N^{\mathrm{PS}}=n\right)$ as the steady state distribution of the number of (non-feedback) customers at the PS node, then it is readily verified that the following decomposition holds:

$$
\mathbb{P}\left(N^{\mathrm{PS}}=n\right)=\xi \pi_{0}(n)+(1-\xi) \pi_{1}(n)
$$

where $\pi_{m}(\cdot)$ is the steady state queue length distribution of the isolated $\mathrm{M}_{\lambda} / \mathrm{G} / 1$ PS queue with $m$ permanent customers, and $\xi$ is the steady state probability that the feedback customer is at the feedback node in the network, i.e., $\pi_{0}(n)=(1-\rho) \rho^{n}, \pi_{1}(n)=(1-\rho)^{2}(n+1) \rho^{n}, \rho:=\lambda \mathbb{E} X$ and $\xi=\mathbb{E} Y /(\mathbb{E} Y+\mathbb{E} Z /(1-\rho))$. 
The result can be extended to multiple feedback customers where the feedback node is a so-called BCMP [3] node. In fact, the feedback node may be replaced by a BCMP network.

\subsection{Multi-class egalitarian processor-sharing with state-dependent capacity}

Consider the egalitarian processor-sharing queue with $K$ customer classes, with the total service capacity dependent on the system state $\mathbf{n}$ through its sum $n_{1}+\cdots+n_{K}(\mathrm{cf}$. [10]). More precisely, $\phi(\mathbf{n})=\varphi(\mathbf{n} \cdot \mathbf{e})$, for all $\mathbf{n} \neq \mathbf{0}$ (null vector), where $\mathbf{e}$ is the vector with 1-entries of appropriate length, $\mathbf{n} \cdot \mathbf{e}$ denotes the inner product, and where $\varphi(\cdot): \mathbb{N} \rightarrow \mathbb{R}_{+}$is an arbitrary positive function. Serving the customers in egalitarian manner reads

$$
\frac{\phi_{i}(\mathbf{n})}{n_{i}}=\frac{\varphi(\mathbf{n} \cdot \mathbf{e})}{\mathbf{n} \cdot \mathbf{e}}, \quad \text { for all } i=1, \ldots, K
$$

and the class capacities $\phi_{i}(\mathbf{n})$ are uniquely characterized and balanced by $\phi_{i}(\mathbf{n})=\Phi\left(\mathbf{n}-\mathbf{e}_{i}\right) / \Phi(\mathbf{n})$, where $\Phi(\mathbf{n})$ is the so-called balance function, and $\mathbf{e}_{i}$ is the $i$ th unity vector of appropriate length (see [12,7]). It is said that the class capacities $\phi_{i}(\mathbf{n})$ are balanced if a function $\Phi(\mathbf{n})$ exists such that $\phi_{i}(\mathbf{n})=\Phi\left(\mathbf{n}-\mathbf{e}_{i}\right) / \Phi(\mathbf{n})$ is satisfied, and equivalently, the class capacities $\phi_{i}(\mathbf{n})$ are balanced if

$$
\frac{\phi_{i}\left(\mathbf{n}-\mathbf{e}_{j}\right)}{\phi_{i}(\mathbf{n})}=\frac{\phi_{j}\left(\mathbf{n}-\mathbf{e}_{i}\right)}{\phi_{j}(\mathbf{n})}, \quad \text { for all } i, j, \text { and } n_{i}>0, n_{j}>0 .
$$

From Eq. (7) and the balance property, we get $\Phi(\mathbf{n})=(\mathbf{n} \cdot \mathbf{e}) ! / \prod_{i=1}^{K} n_{i} !\left(\prod_{j=1}^{\mathbf{n} \cdot \mathbf{e}} \varphi(j)\right)^{-1}$, and without restriction $\varphi(0) \equiv 1$. The joint steady state queue length distribution $\pi(\mathbf{n}):=\mathbb{P}\left(N_{1}=n_{1} ; \cdots ; N_{K}=n_{K}\right)$ is given by the product form:

$$
\pi(\mathbf{n})=(\mathbf{n} \cdot \mathbf{e}) !\left(G \prod_{j=1}^{\mathbf{n} \cdot \mathbf{e}} \varphi(j)\right)^{-1} \prod_{i=1}^{K} \rho_{i}^{n_{i}} / n_{i} !, \quad \text { for } \mathbf{n} \neq \mathbf{0},
$$

with $\rho_{i}=\lambda_{i} \mathbb{E} X_{i}$ and a normalizing constant $G$ (see [7]). It can be shown that the marginal distributions of $\pi(\mathbf{n})$ can be decomposed into queue length distributions of (isolated) permanent customer queues.

Theorem 4. For multi-class egalitarian processor-sharing models, with balanced class capacities $\phi_{k}(\mathbf{n})=\varphi(\mathbf{n} \cdot \mathbf{e}) n_{k} /(\mathbf{n} \cdot \mathbf{e})$, the marginal steady state queue length $N_{k}$ can be decomposed into $N_{k} \stackrel{d}{=} \tilde{N}_{k}^{\left(N_{-k}+1\right) *}$, for all $k=1, \ldots, K$, and where we denote $N_{-k}:=\sum_{i=1, i \neq k}^{K} N_{i}$.

Proof. The decomposition for class $k$ follows from the observation that

$$
\prod_{j=1}^{\mathbf{n} \cdot \mathbf{e}} \varphi(j)^{-1} \equiv\left(\prod_{l=1}^{n_{k}} \varphi\left(l+\left(\mathbf{n} \cdot \mathbf{e}-n_{k}\right)\right)\right)^{-1}\left(\prod_{j=1}^{\mathbf{n} \cdot \mathbf{e}-n_{k}} \varphi(j)\right)^{-1}, \quad \text { for } n_{k} \geq 1,
$$


with $\varphi(0) \equiv 1$. Hence, with (8) and by appropriate summation of $\pi(\mathbf{n})$, the marginal queue length distribution for class $k$ equals:

$$
\begin{aligned}
& \mathbb{P}\left(N_{k}=n_{k}\right) \sim \sum_{\substack{n_{1}, \ldots, n_{k-1} \\
n_{k+1}, \ldots, n_{K}}}(\mathbf{n} \cdot \mathbf{e}) !\left(\prod_{j=1}^{\mathbf{n} \cdot \mathbf{e}} \varphi(j)\right)^{-1} \prod_{i=1}^{K} \frac{\rho_{i}^{n_{i}}}{n_{i} !} \\
& \quad=\sum \frac{(\mathbf{n} \cdot \mathbf{e}) !}{n_{k} !}\left\{\prod_{l=1}^{n_{k}} \varphi\left(l+\left(\mathbf{n} \cdot \mathbf{e}-n_{k}\right)\right)^{-1} \rho_{k}^{n_{k}}\right\}\left(\prod_{j=1}^{\mathbf{n} \cdot \mathbf{e}-n_{k}} \varphi(j)^{-1} \prod_{i \neq k}^{K} \frac{\rho_{i}^{n_{i}}}{n_{i} !}\right),
\end{aligned}
$$

where the symbol $\sim$ denotes equality up to a multiplicative constant. The proof is readily completed, by observing that the expression between parentheses in Eq. (9) is equivalent to the queue length distribution for type $k$ in isolation and with the number of $\mathbf{n} \cdot \mathbf{e}-n_{k}$ permanent customers of the other classes, i.e., $\mathbb{P}\left(\tilde{N}_{k}=n_{k} \mid \mathbf{n} \cdot \mathbf{e}-n_{k}\right.$ permanent customers). The expression after the parentheses in (9) is equivalent to the marginal steady state probability $\mathbb{P}\left(N_{-k}=\mathbf{n} \cdot \mathbf{e}-n_{k}\right)$, after appropriate summation.

\section{Approximation for discriminatory processor-sharing models}

In this section we propose an approximation method for (unconditional) mean sojourn times in generalized discriminatory processor-sharing models (GDPS). The basic approximation assumption in the (G)DPS model is that a class $k$ queue (in isolation) is considered as an egalitarian PS model with (reduced) state-dependent capacity, and where the state-dependent capacity is exogenously determined. In the exact (G)DPS model this is obviously not the case, since the random environments for the different isolated queues in (G)DPS are interrelated and not independent.

We investigate the approximation error if this assumption is made under DPS models. By the exact queue length decomposition results for egalitarian PS models (with state-dependent and balanced class capacities), our method provides exact results if applied on these balanced PS models.

\subsection{General approximation method for mean sojourn times}

For sake of notational convenience, we first consider a two-class GDPS model where the class capacities $\phi_{l}(\mathbf{n})=\phi_{l}\left(n_{1}, n_{2}\right)$ are arbitrary non-negative functions, for $l=1,2$. In addition, we assume a finite number of service positions for both customer types separately $\left(N_{1} \leq m\right.$ and $\left.N_{2} \leq n\right)$, which is not a crucial assumption.

\subsubsection{Approximation method for $K=2$ customer classes}

If one customer type is treated as permanent in the system, then the model is analytically tractable for the non-permanent type. More precisely, the probabilities $\alpha(i, j)$ and $\beta(j, i)$ are easily computed in closed-form by (see [10,17]):

$$
\alpha(i, j) \frac{\rho_{1}^{i} \varphi_{1, i}(j)}{\sum_{k=0}^{m} \rho_{1}^{k} \varphi_{1, k}(j)} \quad \text { and } \quad \beta(j, i)=\frac{\rho_{2}^{j} \varphi_{2, j}(i)}{\sum_{k=0}^{n} \rho_{2}^{k} \varphi_{2, k}(i)},
$$


where

$$
\begin{aligned}
& \varphi_{1, i}(j)=\left(\prod_{k=1}^{i} \phi_{1}(k, j)\right)^{-1}, \quad \varphi_{1,0}(j) \equiv 1 \quad \text { for all } j=0,1, \ldots, n, \\
& \varphi_{2, j}(i)=\left(\prod_{k=1}^{j} \phi_{2}(i, k)\right)^{-1}, \quad \varphi_{2,0}(i) \equiv 1 \quad \text { for all } i=0,1, \ldots, m .
\end{aligned}
$$

Our basic approximation assumption for (G)DPS models is that the linear system given in Corollary 2 is applicable. Under this assumption, we approximate the marginal queue length probabilities $\eta_{i}=\mathbb{P}\left(N_{1}=\right.$ i) and $\xi_{j}=\mathbb{P}\left(N_{2}=j\right)$ by solving the following set of linear equations:

$$
\eta=\mathbf{A} \xi \quad \text { and } \quad \xi=\mathbf{B} \eta
$$

where $\eta=\left(\eta_{0}, \eta_{1}, \ldots, \eta_{m}\right)^{\mathrm{T}}, \xi=\left(\xi_{0}, \xi_{1}, \ldots, \xi_{n}\right)^{\mathrm{T}}$, and the matrices are given by

$$
\mathbf{A}=\left(\begin{array}{cccc}
\alpha(0,0) & \alpha(0,1) & \cdots & \alpha(0, n) \\
\alpha(1,0) & \alpha(1,1) & \cdots & \alpha(1, n) \\
\vdots & \vdots & \ddots & \vdots \\
\alpha(m, 0) & \alpha(m, 1) & \cdots & \alpha(m, n)
\end{array}\right), \quad \mathbf{B}=\left(\begin{array}{cccc}
\beta(0,0) & \beta(0,1) & \cdots & \beta(0, m) \\
\beta(1,0) & \beta(1,1) & \cdots & \beta(1, m) \\
\vdots & \vdots & \ddots & \vdots \\
\beta(n, 0) & \beta(n, 1) & \cdots & \beta(n, m)
\end{array}\right) .
$$

It is not difficult to give conditions such that the (approximated) probability vectors $\eta$ and $\xi$ are uniquely determined after normalization. The system of Eq. (11) is also equivalent to $\eta=(\mathbf{A B}) \eta$, or $\xi=(\mathbf{B A}) \xi$, which can be interpreted as 'solving the equation $\pi=\pi \mathcal{P}$ ', where $\mathcal{P}$ is a transition matrix of a discretetime Markov chain. In many practical (G)DPS models, it is easily verified that the product matrices $(\mathbf{A B})^{\mathrm{T}}$ and $(\mathbf{B A})^{\mathrm{T}}$, have row sums equal to one and do not have negative entries (irreducible, regular stochastic matrices). It is sufficient to have $\phi_{j}(\mathbf{n})>0$ for all $j$, and for all vectors $\mathbf{n}$ with $n_{j}>0$, to guarantee uniqueness of $\eta$ and $\xi$, up to a multiplicative constant.

The approximated (unconditional) mean sojourn time for each class follows from Little's law, and in our case with finite capacity (blocking) we have the approximation:

$$
\mathbb{E} \hat{T}_{1}=\frac{1}{\lambda_{1}\left(1-\eta_{m}\right)} \sum_{i=0}^{m} i \eta_{i} \quad \text { and } \quad \mathbb{E} \hat{T}_{2}=\frac{1}{\lambda_{2}\left(1-\xi_{n}\right)} \sum_{j=0}^{n} j \xi_{j} .
$$

Remark 5. The proposed approximation method is exact for egalitarian PS models with balanced class capacities. The steady state queue length distribution is insensitive to the service time distributions, if and only if the class capacities are balanced (see [7]). Hence, approximations (10)-(12) cannot be exact for PS models with unbalanced class capacities, since the approximation is insensitive to the service time distributions.

\subsubsection{Outline of the approximation method for $K>2$ customer classes}

In principle, our approximation can be applied for general number of customer classes $K$. The method seems very efficient, since only linear systems needs to be solved. However, significantly more compu- 
tational effort needs to be done for increasing $K$. To illustrate the complexity, let us consider the case of $K=3$ classes. Suppose that the class capacities $\phi_{l}(\mathbf{n})=\phi_{l}\left(n_{1}, n_{2}, n_{3}\right), l=1,2,3$, are given in a threeclass GDPS model with system states $\left(N_{1}, N_{2}, N_{3}\right)=(i, j, k)$. The approximated marginal steady state queue length probabilities denoted by $\eta_{i}=\mathbb{P}\left(N_{1}=i\right), \xi_{j}=\mathbb{P}\left(N_{2}=j\right), \zeta_{k}=\mathbb{P}\left(N_{3}=k\right)$ are uniquely obtained from the linear equations (13)-(15), up to a multiplicative constant:

$$
\begin{aligned}
& \eta_{i}=\sum_{j}\left(\sum_{k} \alpha(i \mid j, k) \pi_{3,2}(k \mid j)\right) \xi_{j}=: \sum_{j} a_{i, j} \xi_{j} \\
& \eta_{i}=\sum_{k}\left(\sum_{j} \alpha(i \mid j, k) \pi_{2,3}(j \mid k)\right) \zeta_{k}=: \sum_{k} b_{i, k} \zeta_{k}, \\
& \xi_{j}=\sum_{i}\left(\sum_{k} \beta(j \mid i, k) \pi_{3,1}(k \mid i)\right) \eta_{i}=: \sum_{i} c_{j, i} \eta_{i} \\
& \xi_{j}=\sum_{k}\left(\sum_{i} \beta(j \mid i, k) \pi_{1,3}(i \mid k)\right) \zeta_{k}=: \sum_{k} d_{j, k} \zeta_{k}, \\
& \zeta_{k}=\sum_{i}\left(\sum_{j} \gamma(k \mid i, j) \pi_{2,1}(j \mid i)\right) \eta_{i}=: \sum_{i} e_{k, i} \eta_{i} \\
& \zeta_{k}=\sum_{j}\left(\sum_{i} \gamma(k \mid i, j) \pi_{1,2}(i \mid j)\right) \xi_{j}=: \sum_{j} f_{k, j} \xi_{j} .
\end{aligned}
$$

The coefficients $\alpha(i \mid j, k)$ are given in closed-form formulas, similar to Eq. (10), since $\alpha(\cdot \mid j, k)$ is the steady state queue length distribution for the isolated type 1 queue given that type 2 and 3 customers are permanently in the system. Analogously, the coefficients $\beta(j \mid i, k)$ and $\gamma(k \mid i, j)$ are also easily computed. The pairs of coefficients $\left\{\pi_{2,1}(j \mid i), \pi_{3,1}(k \mid i)\right\},\left\{\pi_{1,2}(i \mid j), \pi_{3,2}(k \mid j)\right\}$, and $\left\{\pi_{2,3}(j \mid k), \pi_{1,3}(i \mid k)\right\}$ are obtained as unique solutions from the linear systems (16)-(18), similar to the approximation method in case of $K=2$, up to multiplicative constant:

$$
\begin{aligned}
& \pi_{2,1}(j \mid i)=\sum_{k} \beta(j \mid i, k) \pi_{3,1}(k \mid i), \quad \pi_{3,1}(k \mid i)=\sum_{j} \gamma(k \mid i, j) \pi_{2,1}(j \mid i) \quad \text { for all } i, \\
& \pi_{1,2}(i \mid j)=\sum_{k} \alpha(i \mid j, k) \pi_{3,2}(k \mid j), \quad \pi_{3,2}(k \mid j)=\sum_{i} \gamma(k \mid i, j) \pi_{1,2}(i \mid j) \quad \text { for all } j \\
& \pi_{1,3}(i \mid k)=\sum_{j} \alpha(i \mid j, k) \pi_{2,3}(j \mid k), \quad \pi_{2,3}(j \mid k)=\sum_{i} \beta(j \mid i, k) \pi_{1,3}(i \mid k) \quad \text { for all } k .
\end{aligned}
$$

The systems (13)-(15) written in matrix form: $\eta=\mathbf{A} \xi, \eta=\mathbf{B} \zeta, \xi=\mathbf{C} \eta, \xi=\mathbf{D} \zeta, \zeta=\mathbf{E} \eta$, and $\zeta=\mathbf{F} \xi$, are efficiently solved by, e.g., the following two systems $\eta=(\mathbf{A C B F D E}) \eta$, and $\xi=(\mathbf{C A D E B F}) \xi$, with normalization $\eta \cdot \mathbf{e}=1$ and $\xi \cdot \mathbf{e}=1$, and where the system for determining $\zeta$ is automatically satisfied and normalized. For increasing $K$, it seems that convenient notation may overcome the increase in complexity.

\subsection{Conservation law}

In this subsection, we obtain a conservation law for unconditional mean sojourn times in a DPS queue, which turns out to be useful in improving the approximations for the lowest priority class. The practical use of a conservation law is that if we are able to obtain accurate approximations 
of $\mathbb{E} T_{k}$ for customer classes $k=1, \ldots, K-1$, then an accurate approximation for class $k$ follows automatically.

Theorem 6. For a K-class DPS queue with fixed capacity, fixed weights, Poisson input, and exponential service requirements with mean $\mathbb{E} X_{i}, i=1, \ldots, K$, the following conservation law for unconditional mean sojourn times holds:

$$
\sum_{j=1}^{K} \rho_{j} \mathbb{E} T_{j}=\sum_{i=1}^{K} \frac{\rho_{i}}{1-\rho} \mathbb{E} X_{i}, \quad \text { independently of }\left(w_{1}, \ldots, w_{K}\right) .
$$

Proof. The result follows from the conservation law [2] for DPS models and from the fact that (1 $\left.F_{j}(x)\right) \mathrm{d} x=\left(1 / \mu_{j}\right) \mathrm{d} F_{j}(x)$ and $\mathbb{E} X_{j}^{2}=2\left(\mathbb{E} X_{j}\right)^{2}=2 / \mu_{j}^{2}$, in case of exponential service requirements.

\subsection{Numerical results}

In this section, we numerically investigate our approximation method with exact results in case of exponential service requirements, for the two- and three-class DPS models with fixed weights and fixed capacity. For numerical experiments and an application of the GDPS model with state-dependent service weights and state-dependent service capacity, we refer to [9].

\subsubsection{Two-class DPS queue}

In the two-class DPS model we refer to type 1 customers as the high priority customers and to type 2 customers as the low priority customers $\left(w_{1}>w_{2}\right)$. In case of exponential service requirements, exact closed-form expressions are given by [11].

The approximated mean sojourn times $\mathbb{E} \hat{T}_{1}$ and $\mathbb{E} \hat{T}_{2}$ are calculated from Eqs. (10)-(12) and with infinite buffer capacity $(m=n=\infty)$. The direct approximation $\mathbb{E} \hat{T}_{2}$ (based on decomposition) for the low priority class can be improved. The improved approximation, denoted by $\mathbb{E} \hat{T}_{2}^{\text {imp }}$, is based on the conservation law (19) and the direct approximation $\mathbb{E} \hat{T}_{1}$ for the high priority class.

Fig. 1 provides graphs for the exact and approximated mean sojourn times for both classes with $\mathbb{E} X_{1}=\mathbb{E} X_{2}=1$, and for different values of $w_{1} / w_{2}$. For class 2 , in addition, the improved approximation $\mathbb{E} \hat{T}_{2}^{\text {imp }}$ is included. Fig. 1 gives results as function of $\rho=\rho_{1}+\rho_{2}$, with $\rho_{1}=\rho_{2}=\rho / 2$. As can be seen from these graphs, the approximation for $\mathbb{E} T_{1}$ is reasonable up to a traffic load $\rho=0.9$ for weight ratios $1 \leq w_{1} / w_{2} \leq 5$. The approximation for $\mathbb{E} T_{2}$ breaks down with increasing difference in weights. However, the approximation $\mathbb{E} \hat{T}_{2}^{\mathrm{imp}}$ that uses $\mathbb{E} \hat{T}_{1}$ to approximate $\mathbb{E} T_{2}$ is accurate for all weight ratios. For a discussion of the quality of the approximation, we refer to Section 4.4.

\subsubsection{Three-class DPS queue}

For the three-class DPS model, we consider the following numerical examples with mean service requirements $\mathbb{E} X_{1}=2$, and $\mathbb{E} X_{2}=\mathbb{E} X_{3}=1$. The exact values for $\mathbb{E} T_{j}, j=1,2,3$, are obtained from [11] as solution of a linear system.

Fig. 2 provides graphs for the exact and approximated mean sojourn times for the three classes and for two sets of weights $w=\left(w_{1}, w_{2}, w_{3}\right)$, respectively for $w=(2,2,1)$ and $w=(3,2,1)$. Fig. 3 provides approximated and exact mean sojourn times for $w=(5,3,1)$ and $w=(10,3,1)$. The approximated mean sojourn times $\mathbb{E} \hat{T}_{j}, j=1,2,3$, are calculated according to the system of Eqs. (13)-(18) and by applying Little's law. The figures are provided as function of the total load $\rho:=\rho_{1}+\rho_{2}+\rho_{3}$, 

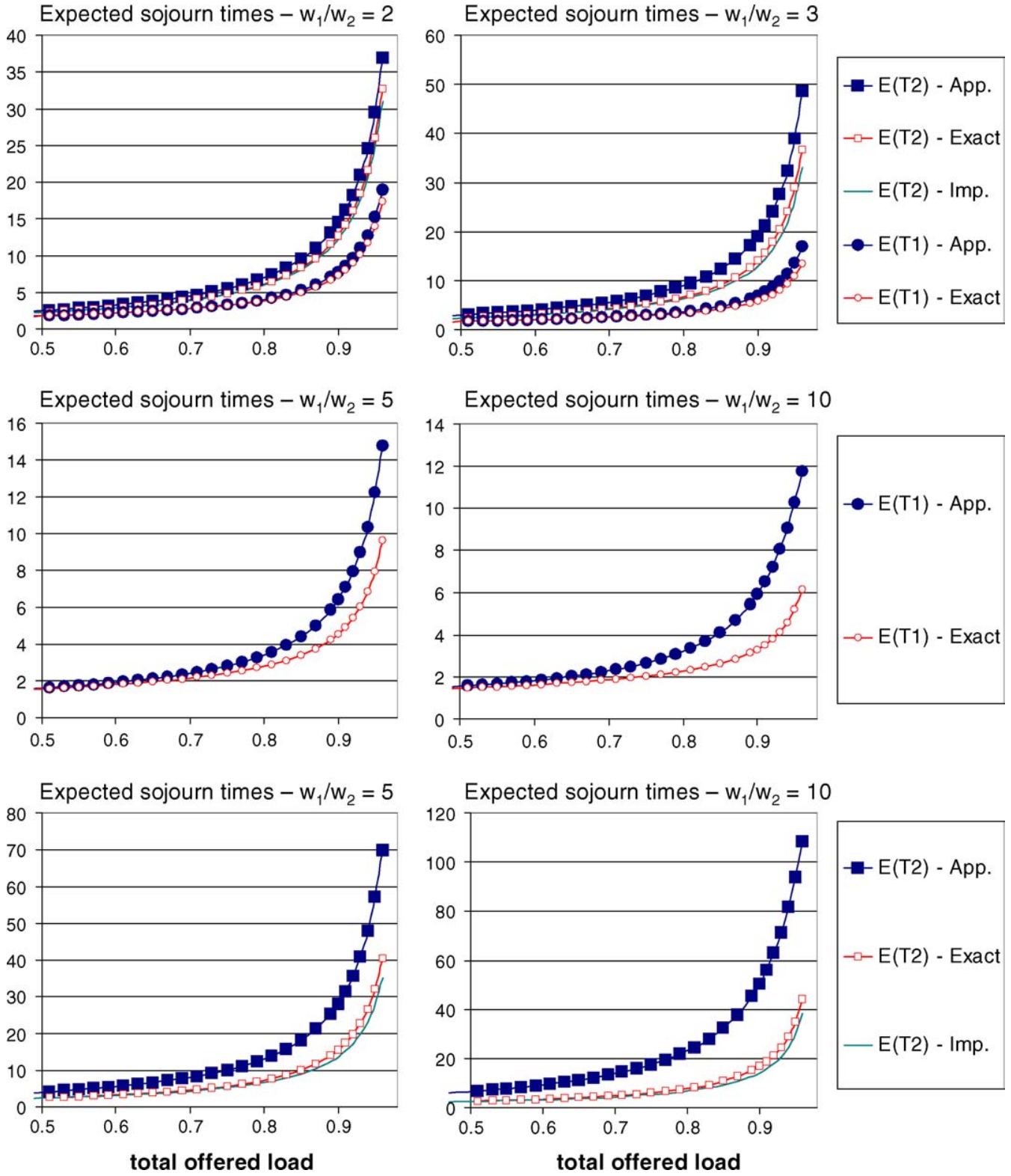

Fig. 1. Exact and approximated mean sojourn times for the two-class M/M/1 DPS queue, with $\mathbb{E} X_{1}=\mathbb{E} X_{2}=1$, and for weight ratios $w_{1} / w_{2} \in\{2,3,5,10\}$.

with $\rho_{1}=\rho_{2}=\rho_{3}=\rho / 3$. In addition, in Fig. 3, an improved approximation $\mathbb{E} \hat{T}_{3}^{\text {imp }}$ is included, based on the conservation law (19) and based on the direct approximations $\mathbb{E} \hat{T}_{1}$ and $\mathbb{E} \hat{T}_{2}$ of the other types.

As can be seen from the graphs (Figs. 2 and 3), the approximations for $\mathbb{E} \hat{T}_{j}$, are accurate as long as the set of weights is 'more or less balanced' (see Remark 5). It seems that our approximation improves for 

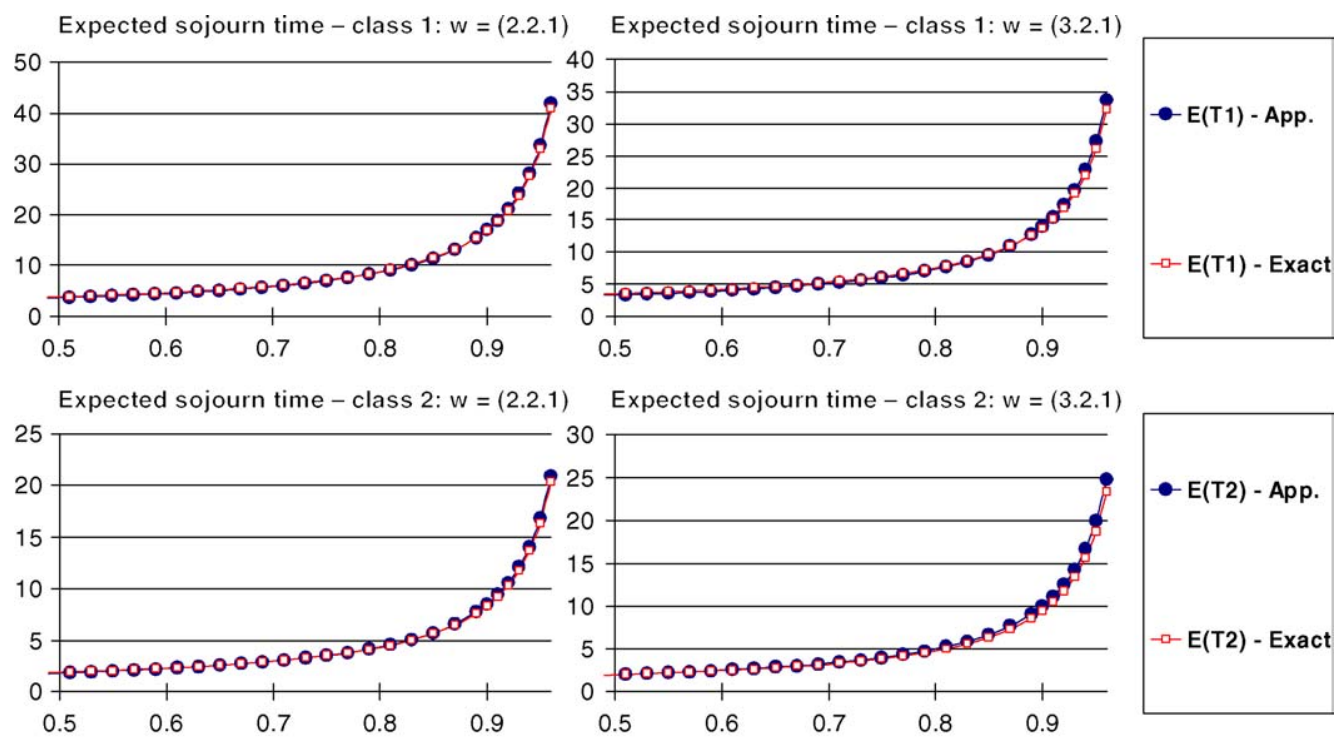

Expected sojourn time - class $3: \mathrm{w}=(2.2 .1)$
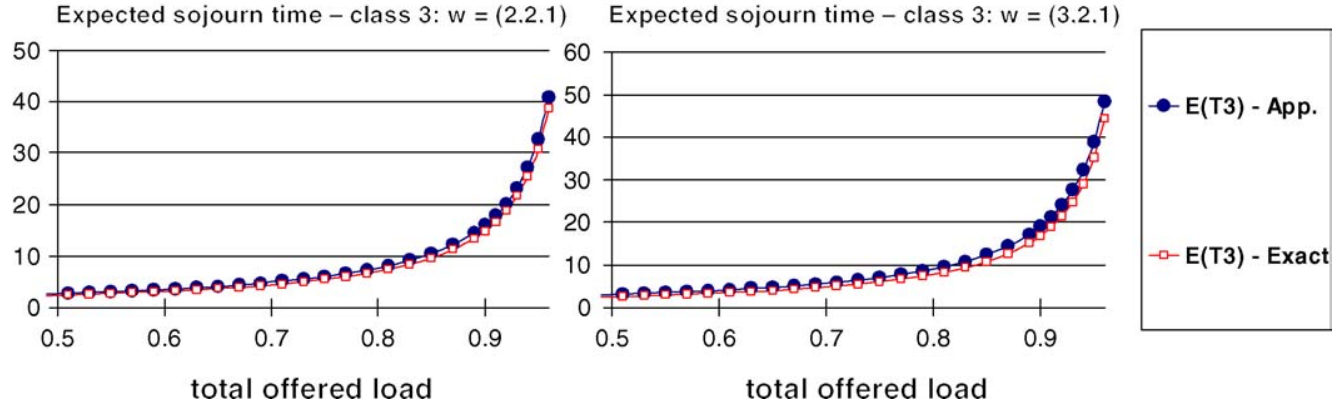

Fig. 2. Exact and approximated mean sojourn times for the three-class M/M/1 DPS queue (with $\mathbb{E} X_{1}=2, \mathbb{E} X_{2}=\mathbb{E} X_{3}=1$ ), for weights $w=(2,2,1)$ and $w=(3,2,1)$.

$K=3$ customer classes. This can be explained by the fact that adding an additional customer class can increase the balance between the classes.

\subsection{Discussion}

In this section, we discuss the quality of our approximation $\mathbb{E} \hat{T}_{j}$ for $\mathbb{E} T_{j}$. In particular, in the case of $K=2$ customer classes, numerical examples indicate that the approximation for the lower priority class $\mathbb{E} \hat{T}_{2}$ is poor when the ratio of weight $w_{1} / w_{2}$ is extremely large (unbalanced), whereas the improved approximation $\mathbb{E} \hat{T}_{2}^{\text {imp }}$ is accurate.

Our basic approximation assumption is that the various customer classes in DPS models are treated as single class egalitarian PS queues with state-dependent (and reduced) service capacity. Supported by the queue length decomposition result for egalitarian PS models, the isolated single class PS queues in a multi-class egalitarian PS queue are exactly related to the other isolated customer queues. 

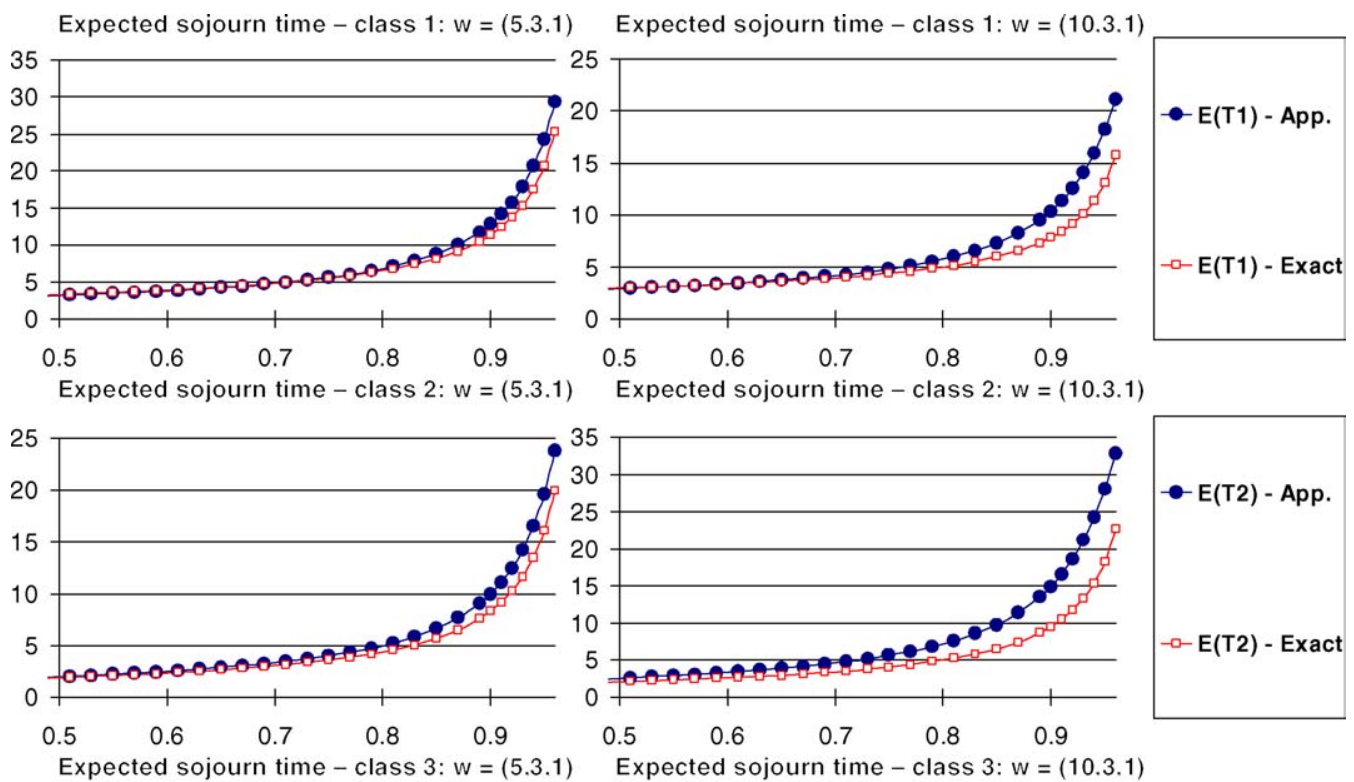

Expected sojourn time - class $3: \mathrm{w}=(5.3 .1) \quad$ Expected sojourn time - class $3: \mathrm{w}=(10.3 .1)$
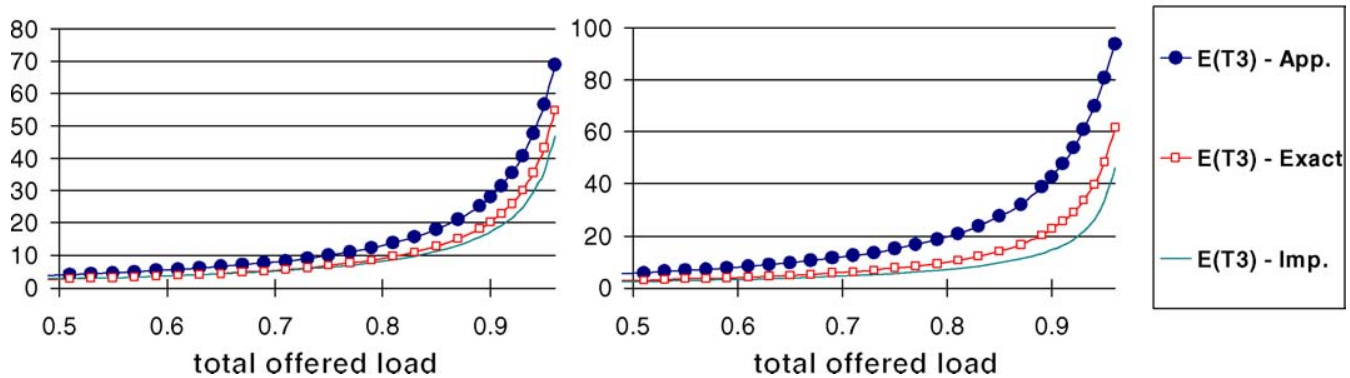

Fig. 3. Exact and approximated mean sojourn times for the 3-class M/M/1 DPS queue (with $\mathbb{E} X_{1}=2, \mathbb{E} X_{2}=\mathbb{E} X_{3}=1$ ), for weights $w=(5,3,1)$ and $w=(10,3,1)$.

When the ratio of weights $w_{1} / w_{2}$ is large, then from a class 2 point-of-view, the queue behaves as an ON-OFF processor-sharing queue [18]. As an illustration, Fig. 4 shows the typical behavior of the queue length processes $N_{i}(t)$ for a two-class DPS queue under heavy load and large ratio $w_{1} / w_{2}$. From a class 2 point-of-view, it seems as if a burst of permanent customers (of size $w_{1} / w_{2}$ ) arrives, when a single customer of type 1 arrives in the original two-class DPS model. Therefore, when the number of class 1 customers gets large enough, then the service process for class 2 may seem frozen (OFF period), and the queue length process for class 2 increases rapidly. However, since the high priority customers (class 1) reside in the system for a relatively short time period (class 1 gets a large share of the capacity), the queue length for the high priority class will decrease rapidly. When there is no high priority customer in the system, the low priority class receives all the available service capacity despite the large ratio of weights $w_{1} / w_{2}$ (ON period), and the queue length for the low priority class decreases significantly.

In the original two-class DPS model, the isolated customer class 2 has a random environment that is severely influenced by the 'burstiness' of class 1 (seen from class 2 point-of-view), while from an 


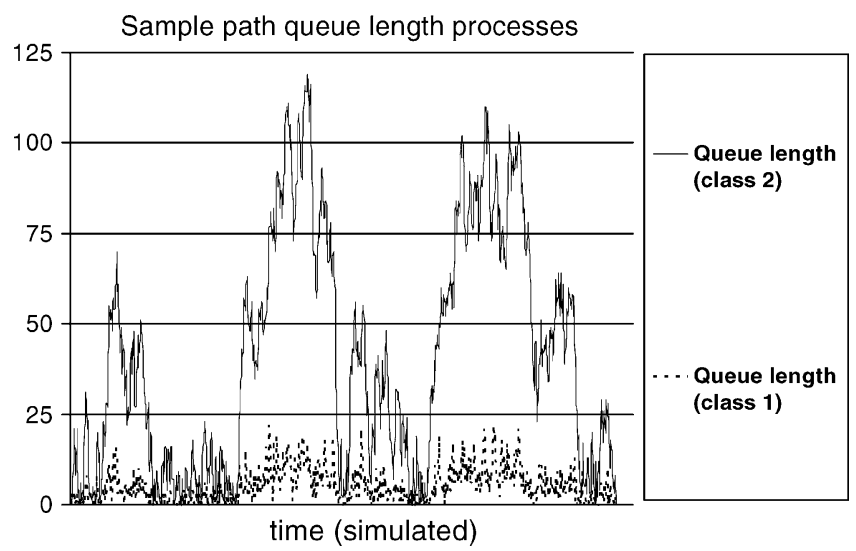

Fig. 4. A sample path of the queue length process $N_{1}(t)$ and $N_{2}(t)$, for a two-class M/M/1 DPS model with $w_{1} / w_{2}=10$, $\lambda_{1}=\lambda_{2}=0.49$, and $\mathbb{E} X_{1}=\mathbb{E} X_{2}=1$.

isolated class 1 point-of-view, it seems as if class 1 behaves according to its own single class and isolated (egalitarian) PS queue, with a random environment that is less fluctuating over time, compared to the isolated class 2 point-of-view.

For the case of $K \geq 3$ customer classes, similar behavior is present in the DPS model. The queue length process of the highest priority class has a significant influence on the queue length process of the lowest priority class, and not in the other way round. However, in the case that more classes are present in the system, with service weights that are in between the highest and lowest priority class, the marginal influence of the highest priority class on the random environment of lowest priority class may be less than in the case of $K=2$.

\section{Conclusion}

In this paper, we obtained a decomposition result for the queue length distributions in the egalitarian processor-sharing models. In particular, for a multi-class egalitarian processor-sharing model, the marginal steady state queue length $N_{k}$ for class $k$, satisfies $N_{k} \stackrel{d}{=} \tilde{N}_{k}^{\left(N_{-k}+1\right) *}$. The latter random variable $\tilde{N}_{k}^{\left(N_{-k}+1\right) *}$ can be interpreted as a random variable denoting the queue length of an isolated processor-sharing queue for class $k$, where the other customer types are permanent customers in the system and $N_{-k}$ represents the random variable of the total number of permanent customers. This result remains valid for egalitarian processor-sharing models with state-dependent system capacity that only depends on the total number of customers.

Motivated by these results, we have proposed an approximation for mean sojourn times in general DPS models. The numerically efficient method is also applicable for GDPS models with state-dependent service capacity and state-dependent service weights. Numerical results have indicated that our approximation is accurate for a wide range of the weight ratios and for moderate loads. The approximation error is small for all loads if the DPS queue has 'nearly balanced' class capacities, which is in agreement with the exact queue length decomposition results. In heavy traffic and for extreme asymmetric weights, the approximation breaks down. However, insights provided in this paper suggest other approximations for 
these regimes, e.g., exploit PS models with ON-OFF periods. This remains a topic for further research. In addition, further theoretical study and improvements of the approximation scheme is desired.

\section{Acknowledgement}

This work has been partially funded by the Dutch Ministry of Economic Affairs under the program ‘Technologische Samenwerking ICT-doorbraakprojecten’, project no. TSIT1025 BEYOND 3G.

\section{References}

[1] E. Altman, T. Jiménez, D. Kofman, DPS queues with stationary ergodic service times and the performance of TCP in overload, Proceedings of IEEE INFOCOM, Hong Kong, 2004.

[2] K. Avrachenkov, U. Ayesta, P. Brown, R. Núñez-Queija, Discriminatory Processor Sharing Revisited, Proceedings of IEEE INFOCOM, Miami, USA, 2005.

[3] F. Baskett, K.M. Chandy, R.R. Muntz, F.G. Palacios, Open Closed, and Mixed Networks of Queues with Different Classes of Customers, J. Assoc. Comput. Mach. 22 (2) (1975) 248-260.

[4] J.V.L. Beckers, I. Hendrawan, R.E. Kooij, R.D. van der Mei, Generalized processor sharing models for Internet access lines, Proceedings of the Ninth IFIP conference on Performance Modeling and Evaluation of ATM and IP Networks, Budapest, 2001.

[5] J.L. van den Berg, Sojourn times in feedback and processor sharing queues, Ph.D. Thesis, Utrecht University, The Netherlands, 1990.

[6] T. Bonald, L. Massoulié, Impact of fairness on Internet performance, Proceedings of the 2001 ACM SIGMETRICS International Conference on Measurement and Modeling of Computer Systems, Cambridge, MA, USA, 2001, pp. 82-91. ACM Press, New York, NY, USA, ISSN: 0163-5999.

[7] T. Bonald, A. Proutière, Insensitivity in processor-sharing networks, Perform. Eval. 49 (2002) 193-209.

[8] S.K. Cheung, J.L. van den Berg, R.J. Boucherie, Insensitive bounds for the moments of the sojourn time distribution in the M/G/1 processor-sharing queue. Memorandum No. 1766. Department of Applied Mathematics, University of Twente, 2005 (available at http://www.math.utwente.nl/publications).

[9] S.K. Cheung, J.L. van den Berg, R.J. Boucherie, R. Litjens, F. Roijers, An analytical packet/flow-level modelling approach for wireless LANs with quality-of-service support, Proceedings of the 19th International Teletraffic Congress, Beijing, China, 2005.

[10] J.W. Cohen, The multiple phase service network with generalized processor sharing, Acta Inform. 12 (1979) $245-284$.

[11] G. Fayolle, I. Mitrani, R. Iasnogorodski, Sharing a processor among many job classes, J. Assoc. Comput. Mach. 27 (1980) 519-532.

[12] F.P. Kelly, Reversibility and Stochastic Networks, Wiley, Chichester, 1979.

[13] G. van Kessel, R. Núñez-Queija, S.C. Borst, Asymptotic regimes and approximations for discriminatory processor sharing, Proceedings of the Workshop on Mathematical Performance Modeling and Analysis, Perform. Eval. Rev. 32 (2004) 44-46 (Special issue on MAMA 2004).

[14] G. van Kessel, R. Núñez-Queija, S.C. Borst, Differentiated bandwidth sharing with disparate flow sizes, Proceedings of IEEE INFOCOM, Miami, USA, 2005.

[15] J. Kim, B. Kim, Sojourn time distribution in the M/M/1 queue with discriminatory processor-sharing, Perform. Eval. 58 (2004) 341-365.

[16] L. Kleinrock, Time-shared systems: a theoretical treatment, J. Assoc. Comput. Mach. 14 (1967) 242-261.

[17] R. Litjens, F. Roijers, J.L. van den Berg, R.J. Boucherie, M. Fleuren, Analysis of flow transfer times in IEEE 802.11 wireless LANs, Ann. Telecommun. 59 (11-12) (2004) 1407-1432.

[18] R. Núñez-Queija, Sojourn times in a processor sharing queue with service interruptions, Queueing Syst. 34 (1-4) (2000) $351-386$. 
[19] K.M. Rege, B. Sengupta, Queue length distribution for the discriminatory processor-sharing queue, Oper. Res. 44 (1996) 653-657.

[20] S.F. Yashkov, Processor-sharing queues: some progress in analysis, Queueing Syst. 2 (1987) 1-17.

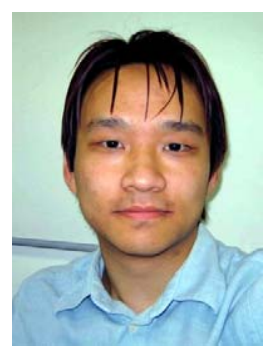

Sing-Kong Cheung received his master's degree in econometrics and operations research from the Vrije Universiteit, Amsterdam in 2002. Currently he is a Ph.D. student at the Department of Applied Mathematics of the University of Twente, The Netherlands. His main research interests are in queueing theory and its applications in performance analysis of communication networks.

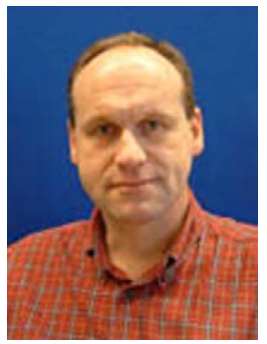

Hans van den Berg received the M.Sc. and Ph.D. degree in mathematics from the University of Utrecht, The Netherlands, in 1986 and 1990, respectively. From 1986, he worked at the Centre for Mathematics and Computer Science (CWI), Amsterdam. In 1990, he joined KPN Research, Leidschendam, The Netherlands, as a member of technical staff in the Department Network Planning. Since 1997, he is a senior research member and is currently leader of the QoS group within TNO Information and Communication Technology, Delft, The Netherlands. He is particularly working on performance modeling, evaluation and dimensioning of fixed and mobile communication networks. In these fields, he has cooperated within many European projects. Since July 2003, he has a part-time full professorship within the Department of Computer Science, University of Twente, The Netherlands.

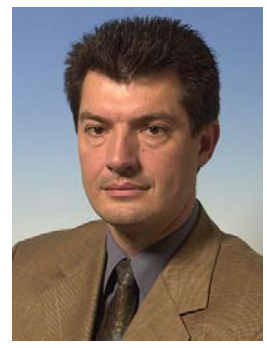

Richard J. Boucherie is full professor of stochastic processes in telecommunications and logistics at the Department of Applied Mathematics of the University of Twente. He studied applied mathematics and theoretical physics at the University of Leiden, and received his Ph.D. from the Vrije Universiteit, Amsterdam in 1992 for a thesis on product-form in queueing networks. Currently, Richards research interests are in stochastic processes such as Petri nets and queueing networks, and their application to capacity allocation and data services in mobile telecommunications systems and optimization in logistics and health care. 